\title{
BMJ Open Point-of-care blood tests during home visits by out-of-hours primary care clinicians; a mixed methods evaluation of a service improvement
}

\author{
Gail Hayward (D) , ${ }^{1}$ Sharon Dixon, ${ }^{1}$ Sophie Garland, ${ }^{2}$ Margaret Glogowska, ${ }^{1}$ \\ Helen Hunt, ${ }^{2}$ Daniel Lasserson (1) ${ }^{3}$
}

To cite: Hayward G, Dixon S, Garland S, et al. Point-of-care blood tests during home visits by out-of-hours primary care clinicians; a mixed methods evaluation of a service improvement. BMJ Open 2020;10:e033428. doi:10.1136/ bmjopen-2019-033428

- Prepublication history for this paper is available online. To view these files, please visit the journal online (http://dx.doi. org/10.1136/bmjopen-2019033428).

Received 04 August 2019 Revised 16 December 2019 Accepted 18 December 2019

Check for updates

(c) Author(s) (or their employer(s)) 2020. Re-use permitted under CC BY-NC. No commercial re-use. See rights and permissions. Published by BMJ.

${ }^{1}$ Nuffield Department of Primary Care Health Sciences, University of Oxford, Oxford, UK

${ }^{2}$ Urgent and Ambulatory Care, Oxford Health NHS Foundation Trust, Oxford, UK

${ }^{3}$ Institute of Applied Health Research, University of Birmingham, Birmingham, UK

Correspondence to

Dr Gail Hayward;

gail.hayward@phc.ox.ac.uk

\section{ABSTRACT}

Objectives We aimed to evaluate test usage and patient and clinician experience following the introduction of point-of-care (POC) blood tests into a primary care out-ofhours service.

Design A mixed methods service evaluation comprising quantitative records of the clinical contexts of tests taken and qualitative interviews with clinicians. Research permissions and governance were obtained for patient interviews.

Setting Out-of-hours primary care.

Participants All patients requiring home visits from the service during the implementation period.

Interventions The i-STAT POC blood test platform was introduced to two bases providing home visits for a period of 8 months. Venous blood samples were used and two cartridges were available. The CHEM8 cartridge measures sodium, potassium, chloride, total carbon dioxide (TCO2), anion gap, ionised calcium, glucose, urea, creatinine, haematocrit and haemoglobin. The CG4 cartridge measures lactate, pH, $\mathrm{PaO} 2$ and $\mathrm{PCO} 2, \mathrm{TCO} 2$, bicarbonate, base excess and oxygen saturation.

Primary and secondary outcome measures The proportion of home visits where tests were taken, the clinical contexts of those tests, the extent to which clinicians felt the tests had influenced their decisions, time taken to perform the test and problems encountered. Clinician and patient experiences of using POC tests.

Results i-STAT POC tests were infrequently used, with successful tests taken at just 47 contacts over 8 months of implementation. The patients interviewed felt that testing had been beneficial for their care. Clinician interviews suggested barriers to POC tests, including practical challenges, concerns about time, doubt over whether they would improve clinical decision making and concern about increased medicolegal risk. Suggestions for improving adoption included sharing learning, adopting a whole team approach and developing protocols for usage.

Conclusions POC tests were not successfully adopted by an out-of-hours home visiting service in Oxfordshire. While some clinicians felt they could not add value, in other cases they resulted in improved patient experience. Adoption could be promoted by improving technical, team and education factors.
Strengths and limitations of this study

- This is the first description of the use of point-ofcare (POC) tests in a UK out-of-hours primary care service.

- The mixed methods approach allowed a nuanced understanding of the impact of POC tests implementation.

- The project was limited to a single service and therefore may lack generalisability to other services.

\section{INTRODUCTION}

Out of hours $(\mathrm{OOH})$ primary care involves high-risk decision-making, as clinicians assess patients with a high prevalence of acute illness without prior knowledge of the patient and with limited access to their background medical information and diagnostic tests. For older patients living with frailty who develop an acute illness, assessments by $\mathrm{OOH}$ clinicians are often made in the patient's home with critical decisions about escalation to hospital care being made with limited evidence. Our previous work on $\mathrm{OOH}$ consultations found that admission due to deterioration after initial assessment was more than twice as likely in patients aged over 80 as those aged under 10, and more likely in patients assessed in their own home. ${ }^{1}$

Delayed escalation to hospital care is associated with greater risk of a poor clinical outcome for many conditions such as serious infections and acute kidney injury. Patients can suffer from prolonged acute illness, incomplete recovery and increased mortality. ${ }^{23}$ Conversely, inappropriate admission to hospital for patients who could be managed in the community is expensive and poses risks from infection and functional decline. ${ }^{45}$

Access to blood tests available rapidly at the point of care (POC) could support $\mathrm{OOH}$ 
clinicians who perform home visits in the early identification of patients with acute illness and in decision making regarding location of care. However, currently, blood POC testing in the home is limited to measurement of blood glucose levels

In a service improvement project, we introduced POC tests giving immediate results from handheld devices into $\mathrm{OOH}$ primary care home visits in Oxfordshire. We employed a mixed methods design to evaluate this service improvement. A quantitative study aimed to describe the usage of tests across the period of implementation. Within this, we embedded a qualitative study to gather information on clinicians' views of having access to the POC tests in this setting and how they were used, and patients' views of the POC tests being offered to them during $\mathrm{OOH}$ home visits.

\section{METHODS \\ Setting}

The Oxfordshire OOH service works out of six bases, two of which were included in this study. The Oxford city base serves an urban population of approximately 160 000, and the Abingdon base serves a more rural population of approximately $130000 .{ }^{6}$ Each base has two cars, so simultaneously two patients can be visited at the same time. In Oxford, mainly GPs perform home visits and in Abingdon typically one GP and one emergency practitioner (EP) perform visits.

\section{Implementing POC blood tests}

The Abingdon and Oxford bases of the Oxfordshire OOH service were each supplied with two Abbott i-STAT devices with a staggered start: Abingdon began on 11
November 2016 and Oxford began on 7 January 2017. Implementation lasted until 2 July 2017.

The i-STAT is a platform POC closed cartridge blood testing system which requires $95 \mu \mathrm{L}$ of blood per cartridge and can be used with capillary, venous or arterial blood. ${ }^{7}$ Two cartridges were available in this project. The Chem8 cartridge measures sodium, potassium, chloride, total carbon dioxide (TCO2), anion gap, ionised calcium, glucose, urea, creatinine, haematocrit and haemoglobin. The CG4 cartridge measures lactate, $\mathrm{pH}, \mathrm{PaO} 2$ and PCO2, TCO2, bicarbonate, base excess and oxygen saturation. At the time of the project, there was no validated portable C Reactive Protein (CRP) test available commercially so this test was not an available option for clinicians. General Practitioners (GPs) and EPs (a role performed by clinicians trained as either emergency nurse practitioners or paramedics) were trained to perform POC tests, with 'champions', who expressed an interest in supporting the intervention, appointed at each base to promote usage and offer additional training. Clinicians were advised to use the tests when they felt it was appropriate to support their decision-making. No guidance on test result interpretation was provided, but EPs could call a GP colleague for advice if needed. On six included shifts, a healthcare assistant (HCA), trained to measure vital signs and perform phlebotomy, accompanied the GPs. This was commenced to support the visiting clinician in performing their assessment, including overcoming any practical barriers to phlebotomy where it was indicated.

Table 1 Test usage across the two sites

\begin{tabular}{lll}
\hline & Abingdon & Oxford \\
\hline Implementation period & $11 / 11 / 16-2 / 7 / 17$ & $7 / 1 / 17-2 / 7 / 17$ \\
\hline Successful blood tests & 48, at 41 contacts & 6, at 6 contacts \\
\hline Failed attempt at blood tests & 11 & 5 \\
Number of potentially appropriate home & 1495 & 833 \\
visits & & \\
Proportion of home visits where successful & $2.7 \%$ & $0.7 \%$ \\
blood test performed & & \\
Type of cartridges used* & 38 Chem8 & 5 Chem8 \\
& 10 CG4 & 1 CG4 \\
Types of clinicians who performed tests & 28 Emergency practitioners & 5 GPs (three with healthcare assistant \\
& 13 GPs (one with healthcare assistant & support) \\
support) & 1 Emergency practitioner \\
Timing of tests & & \\
Overnight 00:00-08:00 hours & 10 & 0 \\
Evening 18:00-00:00 hours & 8 & 1 \\
Daytime† 8:00-18:00 hours & 23 & 5 \\
\hline
\end{tabular}

*The CHEM8 cartridge measures sodium, potassium, chloride, total carbon dioxide (TCO2), anion gap, ionised calcium, glucose, urea, creatinine, haematocrit and haemoglobin. The CG4 cartridge measures lactate, pH, PaO2 and PCO2, TCO2, bicarbonate, base excess and oxygen saturation.

†Daytime contacts were limited to Saturdays, Sundays and public holidays. 
Table 2 Clinical presentations where tests were taken

\begin{tabular}{ll}
\hline Patient problem & Frequency \\
\hline Lower respiratory tract infection & 6 \\
\hline Urinary tract infection & 5 \\
\hline Respiratory condition & 5 \\
\hline Gastroenteritis/vomiting/abdominal pain & 4 \\
\hline Dizziness/collapse/falls & 4 \\
\hline Endocrine & 3 \\
\hline Acute renal failure & 3 \\
\hline Laboratory test abnormal & 2 \\
\hline Dehydration & 2 \\
\hline Sepsis & 2 \\
\hline Heart failure & 2 \\
\hline Malaise and fatigue & 1 \\
\hline Generalised pain & 1 \\
\hline Chest pain & 1 \\
\hline Cellulitis & 1 \\
\hline Musculoskeletal disease & 1 \\
\hline Iron deficiency anaemia & 1 \\
\hline Oedema & 1 \\
\hline Multiple superficial injuries & 1 \\
\hline Terminal illness & 1 \\
\hline
\end{tabular}

\section{Data collection}

\section{Quantitative}

In a service evaluation prospectively approved by Oxford Health NHS Foundation Trust, a member of the direct $\mathrm{OOH}$ care team (SG) used medical records to document types of test used, patient problems and outcomes and timings of consultations in all home visits performed at the Abingdon and Oxford bases. Only routinely collected data were used, and these were anonymised before any analysis was performed. When a test was performed, clinicians used a paper form to document the extent to which they felt that POC tests had influenced their diagnosis on a 5-point scale from extremely likely to extremely unlikely, and recorded time taken for testing and details of unsuccessful test attempts.

\section{Qualitative}

Clinicians: Semistructured qualitative interviews were conducted with clinicians who had access to the POC testing equipment by SD (a GP and researcher). Clinicians were invited to participate by members of the direct care team and the interviews were prospectively approved by Oxford Health NHS Foundation Trust as part of our service evaluation. An information leaflet about the interviews was provided and participants gave informed consent including the right to withdraw and the use of anonymous data and direct quotations in research reports and publications prior to participating.
Patients: Patients who had POC tests performed were provided with an information sheet by the visiting clinician and asked for consent for further contact by the study team. SD or MG (a qualitative researcher) then contacted the patient, explained the study and sent written information. Patients who were willing to participate were offered a choice of face-to-face or telephone interview. Informed consent, including for the audio-recording of the interview, was obtained prior to interview.

\section{Analysis}

Quantitative data were reported descriptively. Home visits for death certification, catheter changes and dressings changes were categorised as unlikely to be appropriate for a POC blood test, the remainder of the home visits were categorised as potentially appropriate and included in the analysis.

Qualitative: The interviews were transcribed in full, entered onto NVivo V.10, and analysed iteratively using thematic analysis. ${ }^{8}$

\section{Patient and public involvement}

The protocol for this project and the patient facing materials were developed following discussion with the Patient and Public Involvement group of the National Institute for Health Research Diagnostic Evidence Cooperative.

\section{RESULTS}

\section{Test usage}

Across both sites, a total of 54 tests were performed at 47 patient contacts. Table 1 details the types of tests, clinicians who took them, timing of tests, failed tests and proportion of home visits where tests were successfully performed, subdivided by site. The majority of tests were performed by the clinicians working from the Abingdon base. In the Oxford base, three of the six home visits where POC tests were used successfully were visits supported by HCAs.

The reasons for failed attempts at blood tests were as follows: Equipment forgotten or out of date (3), unable to bleed patient (3), machine / cartridge fault (5), cartridge filling issue, unable to scan ID, no reason given (2).

\section{Presenting problems of patients receiving blood tests}

The most common presenting problems where a blood test was felt to be appropriate were lower respiratory tract infection and urinary tract infection. The full list of presenting problems is in table 2

\section{Outcomes}

Sixty two per cent of patient contacts where POC tests were used resulted in ongoing care in the community. By comparison, $88.4 \%$ of all home visits where a blood test might have been appropriate resulted in ongoing care in the community. Table 3 illustrates the outcomes of contacts where a POC test was used. Nineteen per cent $(9 / 47)$ of contacts resulted in antibiotic prescriptions being issued by the $\mathrm{OOH}$ clinician, compared to $30 \%$ of the home visits with no POC test performed. 
Table 3 Initial outcomes of contacts where point-of-care (POC) tests performed

\begin{tabular}{ll}
\hline $\begin{array}{l}\text { Initial contact outcome where POC test } \\
\text { performed }\end{array}$ & Frequency \\
\hline Admitted to hospital & 12 \\
Referred to emergency department & 6 \\
Referred to community ambulatory unit & 2 \\
Advised to contact own GP & 22 \\
Own GP to contact patient & 1 \\
No follow-up & 2 \\
Passed to another provider & 2 \\
\hline
\end{tabular}

\section{Workload implications}

In $8 / 28(29 \%)$ of EP contacts where blood was tested, a GP was contacted for advice on the results. Tests took on average $7.3 \mathrm{~min}$. Clinicians rated the extent to which the blood test result was likely to have influenced their diagnosis. Seventeen per cent reported extremely likely, $43 \%$ reported likely and the remaining $40 \%$ reported neutral influence.

\section{Patient experience of POC testing}

Due the frailty inherent within the patient population in this project and the small numbers of tests performed, it proved very difficult to recruit participants who were well enough to consent to interview. We interviewed three patients who had received successful or partially successful POC tests. For these patients, the convenience of having the test at home, for example saving one patient from having to travel a significant distance to an unfamiliar hospital, was invaluable. Patients appreciated results being available immediately, to allow reassurance, or to inform further care decisions.

And we were so grateful for him coming. I thanked him for coming, and he said, "No need to thank, we were concerned. There was a worry, because over the phone we couldn't tell how much you were bleeding. Now, I can tell you, you haven't much.” Patient 1

It would have meant I'd been going on a journey of about 8 miles to a hospital I didn't know, just for a blood test that they weren't really sure was correct or not, you know, to me it was sort of excellent. Patient 2

\section{Clinician perspectives}

We undertook 19 clinician interviews in total: six EPs, of whom three initially trained as paramedics, and three initially trained as emergency nurse practitioners, 1 HCA, 2 interface clinicians (a doctor working in hospital ambulatory care settings) and 10 GP interviews (four GPs interviewed once and three GPs interviewed twice; at the beginning and the end of the study period). The main themes and subthemes are detailed in table 4 .
Table 4 Themes from clinician qualitative interviews

Reasons for low uptake of Time pressures testing

Practical challenges

Uncertain clinical value of testing

Availability of appropriate tests

Potential medicolegal risks

Reasons for differential testing across sites

Factors which could promote test usage

Shared learning and experience of benefits

Potential reasons for differential usage between two out-ofhours sites

Protocols for testing and interpreting

\section{Reasons for low uptake of testing}

In this study, the majority of clinicians did not use POC tests. We interviewed clinicians who had chosen to use them often, rarely and not at all, and highlight the themes arising from the interviews which might help explain the low uptake of testing.

\section{Time pressures}

Clinicians reflected that the time taken to use the tests needed to be considered in the context of the pressures of working in an $\mathrm{OOH}$ setting and to be balanced against the needs of other patients who were still waiting to be seen and how likely the result was to alter the patient's management.

And I'd say it did take at least another ten minutes for this. So, almost doubled my actual consulting time, although you know I guess the counter might be, well if I hadn't done...spent that ten minutes, well actually, calling the hospital or writing a letter, or ringing an ambulance would have taken me a bit longer anyway. GP5

\section{Practical challenges}

Practical challenges, including need to obtain venous blood, apply this to the cartridge, ensure the cartridges were correctly stored and in date, and then interpret the results were all raised as significant considerations. The requirement for venepuncture was identified by many as a barrier. A number of clinicians told us that if the testing had been possible using a finger-prick test, this would have increased their uptake of testing.

I think that the technical side of it is a bit...made it difficult. But as a principle, I think it's very good. For example, as I've said, if you make it a bit easier, like BM [capillary blood glucose] test, that's fantastic GP2

Some clinicians reflected on the challenges of using the equipment in a patients' home, including finding a 
suitable space to use the equipment and whether doing this was appropriate.

it seems fairly unwieldy to use. There's quite a lot of steps involved, and there was a bit of squirting syringes of blood around, which isn't overly appealing GP7

\section{Uncertain clinical value of testing}

Some clinicians expressed the belief that immediate access to blood tests would not their change management or would not add to existing clinical assessment.

The doctors that I... some of the doctors that I spoke to were for it, although some of the older ones tended to think their clinical judgement was...didn't require the i-STAT cartridges at all HCA.

There hasn't been a visit where doing...having access to that would actually change what I do GP7

\section{Availability of appropriate tests}

In this service innovation, clinicians did not have access to some tests commonly used in evaluating acute illnesses, such as CRP. The majority of clinicians we spoke to would have liked to have access to CRP testing to support decision making about both diagnosis and treatment, although others had some concerns. This may have influenced perceptions of likely usefulness and test uptake.

I wish we had a white cell count or a CRP or something like that so that we could definitely tell more for the infection side of it EP1

So, if you have got a high CRP you might be more likely to give antibiotics or not, or that... you know that would also be...guide your treatment GP6

generally the teaching was always don't do a CRP because it's just going to tell you they're unwell and you should know that clinically GP5

\section{Potential medicolegal risks}

Some clinicians reflected that potential medicolegal risks of obtaining and managing abnormal or borderline results were an important consideration for them when deciding whether to use the tests.

And what I think we don't necessarily evaluate is the risk that comes with more information. So, you know you've got a long strip of blood test results, and you've missed the fact that, you know one of them is slightly abnormal but that the patient then deteriorates at a later stage, and is someone going to come back and say, "Well, hang on you missed that." GP5

\section{Potential reasons for differential usage between two $\mathrm{OOH}$ sites}

There was a clear difference in test usage across our two $\mathrm{OOH}$ bases, with the majority of tests performed on visits by staff based in Abingdon. We explored this difference in our analysis. Abingdon is relatively more rural than the city centre Oxford base, meaning that patients would often need to travel further to access tests in a traditional model, whereas some of the GPs working in the Oxford city setting viewed the journey to hospital for blood testing as less inconvenient for patients. A number of clinicians felt that different types of practitioner working in the $\mathrm{OOH}$ setting viewed the POC tests differently, including attitudes and skillsets for taking the blood and using the equipment, and approaches to managing risk and clinical uncertainty. If so, the fact that EPs more commonly worked out of the Abingdon site may have contributed to the differential usage we observed.

I think nursing and paramedic practitioners are a more practical skilled base. So, I think we've been able to get to grips with using it a little bit easier. EP6 I think the GPs are generally, sort of, of the impression as well that, you know oh well...you know my decision's made on my clinical findings with...you know or my examination without needing anything else, or whether that's just a case of, you know they don't...I think that practitioners are more likely to go out and take the bloods, that's probably more to do with it. EP4

I think, as a doctor, we tend to go on more clinical... just how you feel about a patient, Whereas the nurse and paramedics go on definitely protocol and they like to have their numbers. GP6

\section{What factors could have promoted test usage? \\ Shared learning and experience of benefits}

Clinicians identified that learning about where testing had been useful, and sharing those experiences with colleagues, could be a valuable way to learn about and support usage of the equipment. It is possible that the development of positive feedback and a culture that was supportive of usage may have contributed to the differential uptake between sites.

once we start to use it we'll get examples of patients where it's been really helpful. So I think from there people only will then go, "Ah that was a good time to use it," or, "That was useful," and from there people will start to build up and have a place for it. Because I think if people...if initially they don't get an opportunity to use it they might forget, but once they start hearing a little vignette of a case they'll go, "Oh, ah right," and they'll remember the next time. GP 1

I do think once people start to see the benefit to their clinical work from using it that will just spur and encourage them to use it more and more anyway EP1

\section{Team approach to technology adoption}

Making the equipment available as part of a team role, so that it became a normal part of practice when leaving for a visit was identified as valuable.

I'm finding that receptionists are now saying for visits, "Do we need to take the i-STAT?" or hospital at home 
will say, "Do you think we'll take the i-STAT?" or, you know so we're...it's on everyone's mind. GP1

Although some of the GPs were initially apprehensive about having to take responsibility for blood tests done by other practitioners.

what I wouldn't want in the middle of the night is that, you know as practitioner phoning from somewhere saying, "I've seen this patient; I've done all these bloods, this is what they are," and then you say, "Why have you done the bloods?" "Oh just did them to see what they were." GP1

For others, the extra information from POC tests could help the $\mathrm{OOH}$ team members to collectively make decisions about patient care.

I think it's a good. For example if a nurse visits the patient; if you give me the whole...the picture of the patient and give me the vital signs of his or her observation; with the blood result it certainly is very helpful GP2

I'm asking the doctor to make decisions. When he's not seeing the patient and there are obviously, some issues around that as it is. Anything that makes it safe for them to...for us, together, to make a decision, then they're going to be...they'll grab hold of that for sure EP5

\section{Protocols for testing and interpreting}

When considering the training needs of staff, considering how they understand and interpret results, as well as how to use the machine was considered important.

I think all of us maybe need to...especially those of us that are not doctors working in this setting, so a lot of us are nurses and paramedics who have got varying degrees of experience of assessment technique.... but none of us would have really done any blood work and interpretation ourselves. So, I think it's maybe knowing what would be normal in those results and what wouldn't be normal in those results, and what each of maybe the indicator markers very clearly will indicate might be wrong with the patient. EP1

\section{DISCUSSION}

\section{Summary}

$\mathrm{OOH}$ clinicians used POC tests infrequently on home visits, performing tests at just $2 \%$ of all home visits where they may potentially have been of use. Almost two thirds of the tests taken were by EPs and they consulted doctors about the findings in 29\% of cases. Qualitative interviews revealed a number of possible reasons for the very limited usage. These included the practical challenges of using the equipment, balancing the time taken to obtain POC test results against other competing demands, the tests that were available, and perceptions of the risks and added value of the POC tests.

\section{Strengths and limitations}

To our knowledge, this is the first study to describe usage of handheld POC tests in a primary care $\mathrm{OOH}$ service, and to describe clinician and patient experience. Our mixed methods approach gave us a richer understanding of the barriers and facilitators to test usage. The study was limited by the number of $\mathrm{OOH}$ bases available for POC test implementation. Given the variation seen between usage at the two sites, it is unclear whether either setting can provide generalisable results for other services. The study was also limited by the available POC testing platforms which were sufficiently portable to be practical on $\mathrm{OOH}$ home visits. This meant that testing required venous sampling, and could not provide some blood tests, such as CRP, which could be helpful in acute settings. Therefore our findings might not generalise to the use of single biomarker tests or panel tests which use fingerprick testing or offer a different range of blood test results. Indeed our qualitative work suggests that uptake might be improved if technical factors could be overcome in this setting.

Furthermore, due to a lack of training in arterial sampling, the CG4 cartridge was only used with venous blood, when values from arterial samples might have offered additional clinically helpful information. However, there is evidence that venous blood gases can be a useful screen for ventilatory failure in the context of acute illness and can also screen for raised lactate which indicates poorer prognosis in sepsis. ${ }^{9}$ While venous values do not agree with arterial values at abnormal levels, ${ }^{9}$ normal venous values would effectively provide reassurance in a community setting. Finally, it is also possible that the knowledge that usage was being monitored and the requirement for a short feedback form to be completed by clinicians may have affected uptake.

\section{Comparison with other literature}

The limited literature in this area suggests that POC tests could be successfully adopted in $\mathrm{OOH}$ services. Clinicians who provide community care were enthusiastic about the potential benefits of POC blood tests in one interview study. ${ }^{10}$ A Dutch study found that GPs working in $\mathrm{OOH}$ would like greater access to diagnostics, with $93 \%$ of GPs desiring access to POC testing for CRP. ${ }^{11}$ An international survey of GPs also suggested that primary care clinicians want to have access to POC tests to help them with diagnosis and patient care. ${ }^{12}$ Patients have also been shown to be positive about POC testing, reporting that it results in increased confidence in, and a strengthened relationship with, their GP. ${ }^{13}$

A systematic review of qualitative studies exploring clinicians' views on POC testing identified barriers to use including concerns about accuracy, limited situational usefulness, erosion of clinical skills, and costs and time of the equipment. Facilitators included greater diagnostic accuracy, communication with patients and targeted antibiotic usage. ${ }^{14}$ This review included clinicians who had not used POC testing, or who were 
participating in randomised controlled trials, where the use of the equipment was within a trial protocol. In contrast, our qualitative study explored experiences of POC tests when they were simply made available for clinicians to use (or not) at their discretion. The decisionmaking processes described here may therefore offer additional insights into real world implementation.

\section{Implications for research and practice}

The clinical benefits outlined by clinicians who did use POC tests suggest it could add value in urgent care systems if barriers to usage are overcome. Our interviews highlighted technical, team and educational elements which could facilitate adoption of this technology.

Technical elements included the need to minimise the level of practical skill required to take the test and the number of steps in the process of achieving the result. A number of clinicians favoured fingerprick tests, and although the i-STAT can be used with fingerprick samples the volume of blood required makes this challenging to execute. ${ }^{15}$ Improving the range of available blood tests could also improve adoption; some clinicians felt that markers which were not available on this platform such as CRP would have been helpful.

Team elements included the need to establish support systems for learning and maintaining the skills and a team approach to ensuring the equipment was ready for use when visits were planned. Education elements included the need for a forum to share positive impacts and outcomes of testing and clinical scenarios where benefit was likely to be gained. There was an identified need for further training on the implications of test results for EPs, which could have reduced the burden on GP colleagues in the service.

\section{CONCLUSIONS}

The i-STAT POC testing platform was not successfully adopted by an $\mathrm{OOH}$ home visiting service in Oxfordshire. While some clinicians felt they could not add value, in other cases they resulted in improved patient experience. Adoption of POC tests in this context could be promoted by improving technical, team and education factors.

\section{Twitter Daniel Lasserson @DanLasserson}

Contributors GH, DL and HH designed the study. GH led the study and analysed the results. MG and SD designed conducted and analysed the qualitative components. SG and HH oversaw implementation and data collection as part of the direct care team. All authors contributed to the manuscript.

Funding This work was supported by The Health Foundation (grant number 2699). The Health Foundation is an independent charity working to improve the quality of healthcare in the UK. GH was supported by an NIHR Academic Clinical Lectureship and GH and DL were supported by the NIHR Community Healthcare Medtech and IVD Cooperative. The Oxford Academic Health Science Network provided project management and funding support. The views expressed in this publication are those of the authors and not necessarily those of The Health Foundation, the NHS, the NIHR or the Department of Health and Social care.

Competing interests None declared.

Patient consent for publication Not required.

Ethics approval Fulham NRES Committee REC number: 16/L0/1902. provided approval for the patient interviews.

Provenance and peer review Not commissioned; externally peer reviewed. Data availability statement № data are available.

Open access This is an open access article distributed in accordance with the Creative Commons Attribution Non Commercial (CC BY-NC 4.0) license, which permits others to distribute, remix, adapt, build upon this work non-commercially, and license their derivative works on different terms, provided the original work is properly cited, appropriate credit is given, any changes made indicated, and the use is non-commercial. See: http://creativecommons.org/licenses/by-nc/4.0/.

ORCID iDs

Gail Hayward http://orcid.org/0000-0003-0852-627X

Daniel Lasserson http://orcid.org/0000-0001-8274-5580

\section{REFERENCES}

1 Hayward GN, Vincent C, Lasserson DS. Predicting clinical deterioration after initial assessment in out-of-hours primary care: a retrospective service evaluation. Br J Gen Pract 2017;67:e78-85.

2 Silvester KM, Mohammed MA, Harriman P, et al. Timely care for frail older people referred to hospital improves efficiency and reduces mortality without the need for extra resources. Age Ageing 2014;43:472-7.

3 Stewart J, Findlay G, Smith N, et al. Adding insult to injury: a review of the care of patients who died in hospital with a primary diagnosis of acute kidney injury. London: National Confidential Inquiry into Patient Outcome and Death, 2009.

4 Sager MA, Franke T, Inouye SK, et al. Functional outcomes of acute medical illness and hospitalization in older persons. Arch Intern Med 1996;156:645-8.

5 Caplan GA, Sulaiman NS, Mangin DA, et al. A meta-analysis of "hospital in the home". Med J Aust 2012;197:512-9.

6 JSNA Bitesize. Available: https://insight.oxfordshire.gov.uk/cms/ system/files/documents/JSNA_Bitesize_Population_forecasts_ Aug19.pdf

7 Make critical decisions with a single point-of-care testing solution. Available: https://www.pointofcare.abbott/int/en/offerings/istat/istathandheld

8 Strauss A, Corbin J. Basics of qualitative research. Sage publications, 1990.

9 Bloom BM, Grundlingh J, Bestwick JP, et al. The role of venous blood gas in the emergency department: a systematic review and meta-analysis. Eur J Emerg Med 2014;21:81-8.

10 Jones CHD, Glogowska M, Locock L, et al. Embedding new technologies in practice - a normalization process theory study of point of care testing. BMC Health Serv Res 2016;16:591.

11 Schols AMR, Stevens F, Zeijen CGIP, et al. Access to diagnostic tests during GP out-of-hours care: a cross-sectional study of all GP out-of-hours services in the Netherlands. Eur J Gen Pract 2016;22:176-81.

12 Howick J, Cals JWL, Jones C, et al. Current and future use of point-of-care tests in primary care: an international survey in Australia, Belgium, the Netherlands, the UK and the USA. BMJ Open 2014;4:e005611.

13 Laurence CO, Gialamas A, Bubner T, et al. Patient satisfaction with point-of-care testing in general practice. Br J Gen Pract 2010;60:e98-104.

14 Jones CHD, Howick J, Roberts NW, et al. Primary care clinicians' attitudes towards point-of-care blood testing: a systematic review of qualitative studies. BMC Fam Pract 2013;14:117.

15 Dimeski G, Tilley V, Jones BW, et al. Which point-of-care creatinine analyser for radiology: direct comparison of the i-Stat and StatStrip creatinine methods with different sample types. Ann Clin Biochem 2013;50:47-52. 